observed in silicon [A. Hauer and S. J. Burns, Appl. Phys. Lett. 27, 524 (1975)].

${ }^{3}$ B. W. Batterman and H. Cole, Rev. Mod. Phys. $\underline{36}$, 681 (1964).

${ }^{4}$ The effect of thermal vibrations on A.T., and, in general, on dynamical diffraction of $\mathrm{x}$ rays, is well established. The structure factor $F_{H}$ is multiplied by the Debye-Waller factor $e^{-M}$. Experimental proofs are given in the following references: B. Okkerse, Philips Res. Rep. 17, 464 (1962); B. W. Batterman, Phys. Rev. 134, A1354 (1964); H. Wagenfeld and D. Ling, Phys. Lett. 15, 8 (1965); K. Kohra, S. Kikuta, S. Annaka, and S. Nakano, J. Phys. Soc. Jpn. 21, 1565 (1966). An extensive theoretical treatment is given by Y. H. Otsuki, J. Phys. Soc. Jpn. 19, 2285 (1964), and 21, 2300 (1966).

${ }^{5}$ D. L. Spears, Phys. Rev. B $\underline{2}, 1931$ (1970)

${ }^{6}$ E. D. Palik and R. Bray, Phys. Rev. B 3, 3302 (1971).

${ }^{7}$ D. A. O'Connor, Proc. Phys. Soc., London 91, 917 (1967).

${ }^{8} \mathrm{~A}$ CdS transducer was evaporated on the square end of a $5 \times 5 \times 20-\mathrm{mm}^{3}$, dislocation-free silicon crystal, with the long edge parallel to [111]. Longitudinal [111] phonons were piezoelectrically excited at room temperature in CdS by applying an $\mathrm{rf}$ field $(\nu=0.9 \mathrm{GHz})$ by means of a point contact technique. $\mathrm{Cu}-K \alpha$ x-ray radiation $(\lambda=1.54 \AA$ ) was used in this experiment, for which $\mu t \simeq 70$. It was found that the anomalously diffracted beam from the $(2 \overline{2} 0)$ planes was reduced by $16 \%$ when phonons were present. No satellites were found on the wings of the $(2 \overline{2} 0)$ diffraction peak. It was also found that the $(2 \overline{2} 0)$ Bragg case diffraction profile, obtained from the square end opposite to that on which the CdS transducer was evaporated, did not show any effect in the presence of phonons. The latter observation is a further confirmation of the superior sensitivity of anomalous transmission with respect to Bragg case diffraction. This experiment was performed by one of us (R.C.) in collaboration with W. Arnold and A. Freund, at the Max-Planck and the Laue-Langevin Institutes in Grenoble, France.

${ }^{9}$ R. Köhler, W. Möhling, and H. Peibst, Phys. Status Solidi $41,75(1970)$. The same theory can be used for interpreting our results obtained in the Bragg case (see Ref. 1).

\title{
Phase Transition in Surface-Aligned Nematic Films
}

\author{
Ping Sheng \\ RCA Laboratories, Princeton, New Jersey 08540
}

(Received 20 August 1976)

\begin{abstract}
Properties of the nematic-isotropic phase transition in surface-aligned nematic films are calculated using the Landau-de Gennes theory. It is shown that there is a critical thickness $D^{c}$ of the film below which the transition from the nematic phase to the isotropic phase becomes continuous. At the critical thickness the transition is second order and exhibits critical behavior. For N-[e-methoxybenzylidine] - $p$-butylaniline, $D^{c}$ is calculated to be about $1000 \AA$, and the critical temperature is $0.17 \mathrm{~K}$ above the bulk first-order transition temperature. Magnetic and dimensional effects are discussed.
\end{abstract}

Surface alignment ${ }^{1}$ has been widely used to obtain uniform or other desired director configurations in liquid-crystal cells made for display or measurement purposes. There are various alignment techniques involving different treatments of the solid surface such as chemical etching, ${ }^{2}$ coating with lecithin, ${ }^{3}$ adsorption of organic surfactant additives, ${ }^{4}$ unidirectional rubbing, ${ }^{5}$ or the oblique evaporation method. ${ }^{6}$ However, regardless of the technique, in the uniform parallel or perpendicular orientation (as opposed to random parallel orientation) surface alignment always produces an ordered layer at the solid-liquid crystal interface. This order is then transmitted to the bulk liquid crystal by elastic forces. In this Letter, I use the Landau-de Gennes theory to calculate the properties of the nematic-isotropic phase transition in surface-aligned nematic films. It is shown that there exists a critical thickness of the film, of the order of $1000 \AA$ for typical liquid crystals, below which the transition is continuous. At the critical thickness the transition is second order and exhibits critical behavior.

Consider a nematic film of uniform thickness $D$ with its normal along the $z$ axis. The solid-liquid crystal interface is defined as $z=0$, and the free surface of the film is at $z=D$. The microscopic order parameter ${ }^{7} S$ of the nematic liquid crystal, with its value lying between 0 and 1 , is defined as the spatial average of $P_{2}(\cos \theta)$, where $\theta$ is the angle between the long axis of the molecule and the director, and $P_{2}$ denotes Legendre polynominal of second order. The nematic director has to be uniform throughout the film since one surface is free. In terms of the order parameter, the Landau-de Gennes free-energy density $\varphi$ for uniform 
director configuration is given $b^{7}$

$$
\begin{aligned}
& \varphi=\varphi_{0}+f(S)+L(\nabla S)^{2}, \\
& f(S)=a\left(T-T^{*}\right) S^{2}+B S^{3}+C S^{4},
\end{aligned}
$$

where $T$ is the temperature, $\varphi_{0}$ is a constant, and $a, T^{*}, B, C$, and $L$ are material parameters which can be determined from thermodynamic properties and fluctuation phenomena of the nematic liquid crystal. In Eq. (2), the power of the quantity $\left(T-T^{*}\right)$ is assumed to be 1 since there is evidence that this gives the correct pretransitional behavior. ${ }^{8}$ At $z=0$, the chemical or physical nature of the treated solid surface defines a preferred direction of alignment for nematic molecules. ${ }^{9}$ This substrate-alignment effect, the magnitude of which depends on the strength of substrate-liquid crystal interaction, serves to produce an ordered layer of liquid crystal molecules at the interface. For simplicity, in the following we consider the case where the nematic molecules are strongly anchored to the solid substrate (such as by silane coupling agents) with $S$ $=1$ at the interface. ${ }^{10}$ At finite $z$ the thermal equilibrium value of $S(z)$ is obtained by minimizing the total free energy $\Phi$ of the system, given as the volume integral of $\varphi$. The minimization of $\Phi$ with respect to the functional form of $S(z)$ requires that the correct $S(z)$ satisfies the Euler equation, $\partial \varphi / \partial S=\nabla \cdot[\partial \varphi / \partial(\nabla S)]$. Since the system is uniform in the $x$ and $y$ directions, the Euler equation can be integrated once to obtain

$$
L(\boldsymbol{d} S / \boldsymbol{d} z)^{2}=f(S)+K,
$$

where the constant $K$ can be determined by observing that, since $z=D$ is a free surface, the transversality condition ${ }^{11}$ of variable endpoint problems in variational calculus requires

$$
d S /\left.d z\right|_{z=D}=0 .
$$

Combining Eqs. (3) and (4) yields

$$
\xi_{0}^{2}(d S / d z)^{2}=F(S)-F\left(S_{b}\right),
$$

where $\xi_{0} \equiv\left(L / a T_{K}{ }^{0}\right)^{1 / 2}$ is the correlation length below which there can be no large variation in the value of $S, T_{K}{ }^{0}$ is the bulk first-order transition temperature, $F(S) \equiv f(S) / a T_{K}{ }^{0}$, and $S_{b}$ is the value of $S$ at the free surface $z=D$.

For a given film thickness $D$, the value of $S_{b}$ can be determined from Eq. (5) as

$$
\frac{D}{\xi_{0}}=\int_{S_{b}}^{1} \frac{d s}{\left[F(S)-F\left(S_{b}\right)\right]^{1 / 2}} .
$$

The corresponding free energy of the system is given by

$$
\frac{\Phi}{A a T_{K}^{0} \xi_{0}}=\frac{D}{\xi_{0}} F\left(S_{b}\right)+2 \int_{S_{b}}^{1}\left[F(S)-F\left(S_{b}\right)\right]^{1 / 2} d S,
$$

where $A$ is the area of the sample. When there is more than one solution to Eq. (6), the results must be substituted into Eq. (7) to select the one solution that gives the lowest free energy. Once $S_{b}$ is determined, $S(z)$ can be calculated from the equation

$$
\frac{z}{\xi_{0}}=\int_{S(z)}^{1} \frac{d s}{\left[F(S)-F\left(S_{b}\right)\right]^{1 / 2}} .
$$

In the special case of $D=\infty$, Equation (7) gives the free energy density of the system as $\Phi / V$ $=a T_{K}{ }^{0} F\left(S_{b}\right)$ and the solution of Eq. (6) yields values of $S_{b}$ that are the locations of local extrema of $F(S)$. This is obvious since the integral of Eq. (6) diverges logarithmically when $F^{\prime}\left(S_{b}\right)=0$. The value of $S_{b}$ in this case, as required by the minimization of free energy, is therefore given by the location of the absolute minimum of $F(S)$. We can thus recover the expected bulk behavior from Eqs. (6) and (7).

When the film has a finite thickness, the calculation is carried out numerically. In the following I will use the measured values ${ }^{12}$ of $a=0.045 \mathrm{~J}$ $\mathrm{cm}^{-3} \mathrm{~K}^{-1}, B=0.197 \mathrm{~J} \mathrm{~cm}^{-3}, C=0.307 \mathrm{~J} \mathrm{~cm}^{-3}, T^{*}$ $=318.3 \mathrm{~K}$, and $T_{K}{ }^{0}=319 \mathrm{~K}$ for $\mathrm{N}-(p-$ methoxybenzylidine]-p-butylaniline (MBBA) in my calculation. Figure 1 gives the variation of $S_{b}$ as a function of temperature for various film thicknesses. It can be seen that significant modification of the phase transition occurs when the film thickness is below $150 \xi_{0}$. There is a critical thickness, in this

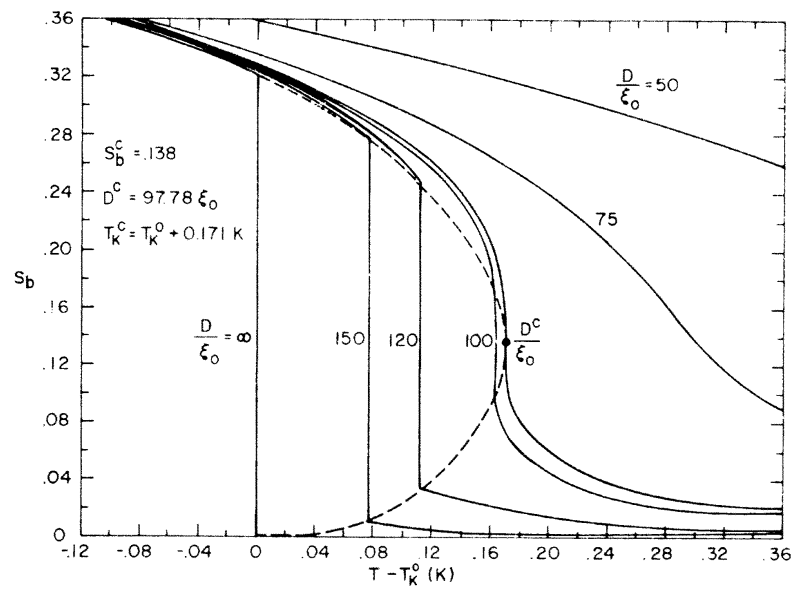

FIG. 1. The variation of $S_{b}$ as a function of temperature for different film thicknesses. The dashed line is the coexistence curve. 


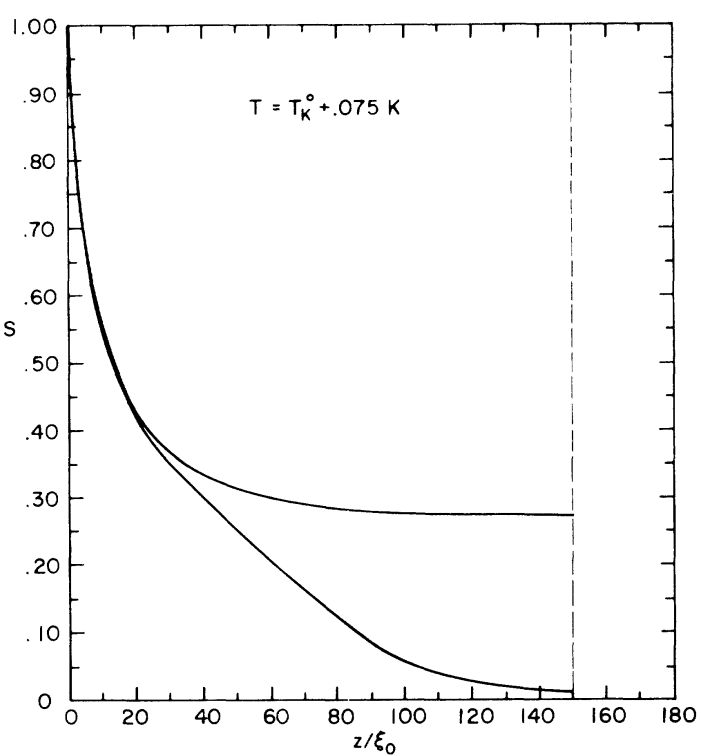

FIG. 2. The variation of $S$ as a function of $z$ for $D$ $=150 \xi_{0}$. The lower curve shows $S(z)$ just above the transition temperature and the upper curve shows $S(z)$ just below the transition temperature.

case $D^{c}=97.78 \xi_{0}$, below which the transition is continuous. Since $\xi_{0}$ is typically ${ }^{13} 10 \AA, D^{c}$ is therefore in the order of $1000 \AA$. At the critical thickness the transition is second order with a critical temperature $T_{K}{ }^{c}=T_{K}{ }^{0}+0.171 \mathrm{~K}$. Near the critical point the temperature dependence of $S_{b}$ is found to be $\left(S_{b}-S_{b}{ }^{c}\right) \propto\left(T-T_{K}{ }^{c}\right)^{1 / 3}$, where $S_{b}{ }^{c}$ $=0.138$. The cirtical point shown in Fig. 1 is somewhat similar to the gas-liquid critical point. However, there is an important difference in that the law of rectilinear diameter is not satisfied for the present case. ${ }^{14}$ The variation of the order parameter as a function of $z$ is shown in Fig. 2 for the case of $D=150 \xi_{0}$ at the transition temperature. The two curves show $S(z)$ just before and after the transition has occurred.

The specific heat $C$ of the system can be obtained directly from Eq. (7):

$$
\begin{aligned}
& \frac{C}{A}=\left.\frac{T}{A} \frac{\partial^{2} \Phi}{\partial T^{2}}\right|_{D \text { const }}=a \xi_{0} \frac{T}{2 T_{K}^{0}}\left[I_{1}+\frac{1}{2} F^{\prime}\left(S_{b}\right) \frac{I_{2}^{2}}{J}\right] \\
& I_{1}=\int_{S_{b}}^{1} \frac{\left(S^{2}-S_{b}{ }^{2}\right)^{2} d S}{\left[F(S)-F\left(S_{b}\right)\right]^{3 / 2}} \\
& I_{2}=\int_{S_{b}}^{1} \frac{\left(S^{2}-S_{b}{ }^{2}\right) d S}{\left[F(S)-F\left(S_{b}\right)\right]^{3 / 2}} \\
& J=\left[F(1)-F\left(S_{b}\right)\right]^{-1 / 2} \\
& \quad+\frac{1}{2} \int_{S_{b}}^{1} \frac{F^{\prime}(S)-F^{\prime}\left(S_{b}\right)}{\left[F(S)-F\left(S_{b}\right)\right]^{3 / 2}} d S
\end{aligned}
$$

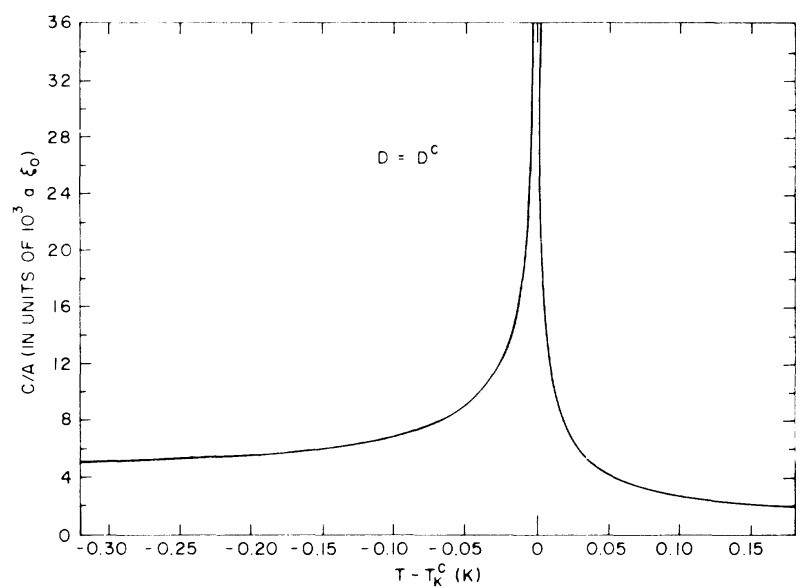

FIG. 3. The variation of the specific heat per unit area as a function of temperature. The divergence at $T=T_{K}^{c}$ is proportional to $\left|T-T_{K}{ }^{c}\right|^{-2 / 3}$.

In Fig. 3 the specific heat is plotted as a function of temperature for $D=D^{c}$. It is calculated that $C$ diverges at the critical temperature as $C \sim \mid T$ $-\left.T_{K}{ }^{c}\right|^{-2 / 3}$. If the temperature is fixed at $T_{K}{ }^{c}$, the divergence of $C$ as a function of film thickness is found to be $\left|D-D^{c}\right|^{-2 / 3}$.

The magnetic effects can be similarly calculated by including the term $\mathrm{m}^{7}-\Delta \chi S H^{2 / 3}$ in $f(S)$, Eq. (2). Here $H$ is the strength of the magnetic field along the nematic director and $\Delta \chi$ is the diamagnetic anisotropy. Calculation shows that $d S_{b} / d H^{2}$ diverges as $\left|T-T_{K}^{c}\right|^{-2 / 3}$ for $\boldsymbol{D}=D^{c}$. Since the Cotton-Mouton coefficient $C_{M}$ for the magnetic birefringence effect can be expressed as a function of $d S_{b} / d H^{2}$, it is expected that $C_{M}$ would similarly diverge. Calculation also shows that $D^{c}$, $T_{K}^{c}$, and $S^{c}$ are monotonically increasing functions of $H$. Therefore, the critical point in Fig. 1 is actually the endpoint of a line of critical points in the $H-D-T$ space. For a sufficiently strong magnetic field, of the order of $10^{6}$ Oe for MBBA, $D^{c}$ diverges. In this case one has a critical point in the $H-T$ surface for bulk liquid crystal, ${ }^{15}$ and it can be shown there that the coexistence curve satisfies the law of rectilinear diameter.

It is well known that the values of the critical exponents in real materials differ from those predicted by the Landau or mean-field-type theories. ${ }^{16}$ In the present case although there is evidence that the Landau-de Gennes theory gives the correct exponent behavior for pretransitional phenomena in nematic-isotropic phase transitions, ${ }^{8}$ the lack of exact correspondence between the present critical point and other known critical points 
precludes a ready prediction for the values of the critical exponents in real situations. Moreover, it has been recognized that the values of the critical exponents are dependent on the dimensionality of the system. ${ }^{16}$ For the nematic film at $D=D^{c}$, it is reasonable to expect that at temperatures far away from $T_{K}{ }^{c}$ the correlation length of order parameter fluctuations is small compared to $D^{c}$, and therefore the system exhibits three-dimensional behavior. However, when $T$ is sufficiently close to $T_{K}{ }^{c}$, the correlation length can exceed $D^{c}$, and the system should exhibit two-dimensional critical behavior. It follows that there is a transition from three-dimensional behavior to two-dimensional behavior as the critical point is approached. Under the assumption that the critical length grows as $\xi_{0}\left|T-T_{K}\right|^{-1 / 2}$ and $\xi_{0} \simeq 10 \mathrm{~A}$, such a transition is expected to occur at about $0.01 \mathrm{~K}$ from $T_{K}{ }^{c}$. Experimental investigation of these predicted phenomena would be not only interesting in itself but also helpful in providing further insights into cooperative effects in thin nematic films and the nature of solid-liquid crystal interaction.

${ }^{1} \mathrm{~L} . \mathrm{A}$. Goodman, in Introduction to Liquid Crystals, edited by E. B. Priestley, P. J. Wojtowicz, and P. Sheng (Plenum, New York, 1975), p. 219.

${ }^{2}$ M. F. Schiekel and K. Fahrenschon, Appl. Phys.
Lett。19, 391 (1971).

${ }^{3} \mathrm{~J}$. F. Dryer, in Liquid Crystals 3 , edited by G. Brown and M. Labes (Gordon and Breach, London, 1971), p. 1113.

${ }^{4}$ W. Haas, J. Adams, and J. Flannery, Phys. Rev. Lett. 25, 1326 (1970)。

${ }^{5}$ H. Zocher and K. Coper, Z. Phys. Chem. 132, 195 (1928)。

${ }^{6}$ J. L. Janning, Appl. Phys. Lett. 21, 173 (1972).

${ }^{7}$ P. G. de Gennes, Mol. Cryst. Liq. Cryst. 12, 193 (1971).

${ }^{8} \mathrm{~T}$.W. Stinson and J. D. Litster, Phys. Rev. Lett. $\underline{25}$, 503 (1970).

${ }^{9} \mathrm{P}$. G. de Gennes, The Physics of Liquid Crystals (Clarendon, Oxford, 1974), p. 70-79.

${ }^{10}$ The results of calculation are insensitive to the exact value of $S$ at the interface, $z=0$, as long as it is in the range of 0.5 to 1 . For example, if $S(z=0)=0.5$, then $D^{c}$ is decreased by about $10 \xi_{0}$ from that of $S(z=0)$ $=1$.

${ }^{11}$ See, for example, J. Irving and N. Mullineaux, Mathematics in Physics and Engineering (Academic, New York, 1959), p. 374。

${ }^{12}$ Y. Poggi, J。C. Filippini, and E. Aleonard, Phys. Lett。57A, 53 (1976).

${ }^{13}$ T. W. Stinson and J. D. Litster, Phys. Rev. Lett. $\underline{30}$, 688 (1973).

${ }^{14} \mathrm{~J}$. S. Rowlinson, Liquids and Liquid Mixtures (Butterworth Scientific Publications, London, 1959), p. 91.

${ }^{15}$ P. J. Wojtowicz and P. Sheng, Phys. Lett. $\underline{48 \mathrm{~A},} 235$ (1974).

${ }^{16} \mathrm{H}$. E. Stanley, Introduction to Phase Transitions and Critical Phenomena (Oxford Univ. Press, New York, 1971).

\title{
Theory of Vibrational Properties of Amorphous Alloys: Application to $\mathrm{Si}_{x} \mathrm{Ge}_{1-x}$
}

\author{
Felix Yndurain \\ Department of Physics, * University of California, Berkeley, California 94720
}

(Received 27 May 1976)

\begin{abstract}
The phonon density of states of amporphous $\mathrm{Si}_{x} \mathrm{Ge}_{1-x}$ alloys has been calculated for the entire range of concentrations. My calculation is based on the cluster Bethe lattice approximation in which the Bethe lattice leaves out everything but the short-range order. Effects of like-atom and unlike-atom bonds are studied. Localized resonant and extended states are identified.
\end{abstract}

The study of the vibrational properties of noncrystalline materials - amorphous semiconductors, ${ }^{1}$ glasses, ${ }^{2}$ mixed-crystals, ${ }^{3}$ and alloys ${ }^{3,4-i s}$ of great current interest. The main experimental tool, Raman scattering, gives information about phonon density of states in the entire frequency range. ${ }^{5}$ As an example, analysis of Raman spectra has identified the presence of homonuclear bonds ${ }^{6}$ in amorphous $\mathrm{SiC}$. It has also been used to obtain information on coordination, topological arrangement, and bonding character of some chalcogenide glasses, ${ }^{1,7,8}$ as well as local modes in covalent crystalline semiconducting alloys. ${ }^{9}$ Finally, it has been used to obtain information about the phonon density of states of amorphous semiconductors ${ }^{1,10}$ and their alloys. ${ }^{11}$

In spite of the richness of the experimental data, the theoretical situation is not very satisfactory. Most of the work ${ }^{12,13}$ is concerned with homopolar amorphous semiconductors. 